\title{
Влияние глубоких центров на конфайнмент носителей в квантовых ямах In GaN/GaN и эффективность светодиодов
}

\author{
(С) Н.И. Бочкарева, Ю.Г. Шретер \\ Физико-технический институт им. А.Ф. Иофрфе Российской академии наук, \\ 194021 Санкт-Петербург, Россия \\ ฯ E-mail: y.shreter@mail.ioffe.ru
}

(Получена 7 декабря 2017 г. Принята к печати 27 декабря 2017 г.)

\begin{abstract}
Туннелирование электронов с участием глубоких центров в светодиодных $p-n$-структурах с квантовыми ямами InGaN/GaN снижает эффективную высоту инжекционного барьера, но приводит к зависимости эффективности излучения от плотности и энергетического спектра дефектов в GaN. При прыжковой проводимости через область пространственного заряда основная часть прямого напряжения падает у границы с квантовой ямой, где плотность глубоких состояний на квазиуровне Ферми наименьшая. В результате у границы происходит спрямление изгиба зон, а с ростом тока и изменение направления электрического поля, что приводит к ослаблению конфайнмента дырок, их безызлучательной рекомбинации в $n$-барьере и падению эффективности. Низкая эффективность зеленых $\mathrm{GaN}$-светодиодов связывается с доминированием глубоких центров и недостаточной плотностью мелких центров в энергетическом спектре дефектов в барьерных слоях вблизи границ с квантовой ямой. Предложенная модель подтверждается ступенчатым характером экспериментальных зависимостей тока, емкости и эффективности зеленых и синих светодиодов от прямого смещения, отражающих вклад центров окраски, ответственных за полосы дефектной фотолюминесценции в $\mathrm{GaN}$.
\end{abstract}

DOI: 10.21883/FTP.2018.07.46055.8790

\section{1. Введение}

Эффективность излучения квантовых ям InGaN/GaN быстро уменьшается при повышении концентрации индия и увеличении длины волны излучения в зеленой области спектра [1-3]. Эта проблема, известная как проблема зеленой долины (green gap problem), наряду с проблемой падения эффективности излучения квантовых ям In $\mathrm{GaN} / \mathrm{GaN}$ с ростом уровня накачки (efficiency droop problem) интенсивно изучается в течение последних двух десятилетий, но физическая природа этих эффектов до сих пор остается предметом дискуссий, а возможность решения этих проблем неопределенной.

Низкая эффективность $\mathrm{GaN}$-светодиодов при повышении концентрации индия в активной области связывается с проявлением различных факторов, включая увеличение поляризационного поля в квантовой яме [4], увеличение флуктуаций концентрации индия [5], более высокую плотность дислокаций [2] и точечных дефектов [6]. Одним из основных подходов, принятых в литературе для выяснения причин, влияющих на эффективность, остается анализ формы экспериментальной зависимости эффективности от уровня инжекции. Предполагается, что эффективность определяется излучательной и безызлучательной рекомбинацией в квантовой яме InGaN/GaN. Между тем в светодиодных $p$-n-структурах с квантовыми ямами $\mathrm{InGaN} / \mathrm{GaN}$ доминирует туннельный механизм прохождения тока [7-10]. Высокая туннельная прозрачность барьеров ослабляет конфайнмент носителей в квантовой яме и может оказывать значительное влияние на эффективность излучения.
Туннелирование носителей облегчается присутствием глубоких центров. Высокая плотность и широкий энергетический спектр глубоких центров обнаруживаются в $\mathrm{GaN}$ оптическими методами: фотопроводимости [11], фотоемкости [12], оптического поглощения [13-16], фотолюминесценции (см. обзор [17]). В работах $[18,19]$ предполагается, что туннелирование носителей в барьерных структурах на основе $\mathrm{GaN}$ происходит с участием центров окраски, ответственных за полосы дефектной фотолюминесценции в $\mathrm{GaN}$.

Цель данной работы - изучение механизма влияния глубоких центров в $\mathrm{GaN}$ на распределение поля в области пространственного заряда (ОПЗ) $p$-n-структур с квантовыми ямами InGaN/GaN, определяющего в свою очередь конфайнмент носителей в квантовой яме $\mathrm{InGaN} / \mathrm{GaN}$ и эффективность $\mathrm{GaN}$-светодиодов. C этой целью в работе изучаются общие для зеленых и синих светодиодов особенности поведения тока, емкости, эффективности при сканировании квазиуровнями Ферми энергетического распределения глубоких центров в прямосмещенных $p-n$-структурах светодиодов. Результаты сопоставляются с результатами измерений энергетического спектра дефектов в слоях $\mathrm{GaN}$ методами фотолюминесценции и оптической плотности.

\section{2. Эксперимент}

Для детальных исследований в работе были выбраны коммерческие планарные светодиоды Nichia с одиночной квантовой ямой $p$-AlGaN/ $\operatorname{In}_{x} \mathrm{Ga}_{1-x} \mathrm{~N} / n-\mathrm{GaN}$ толщиной $30 \AA[20]$, пиковой энергией излучения $h v_{p}=2.46$ и 2.34 эВ (зеленые диоды А и В соответственно) и 


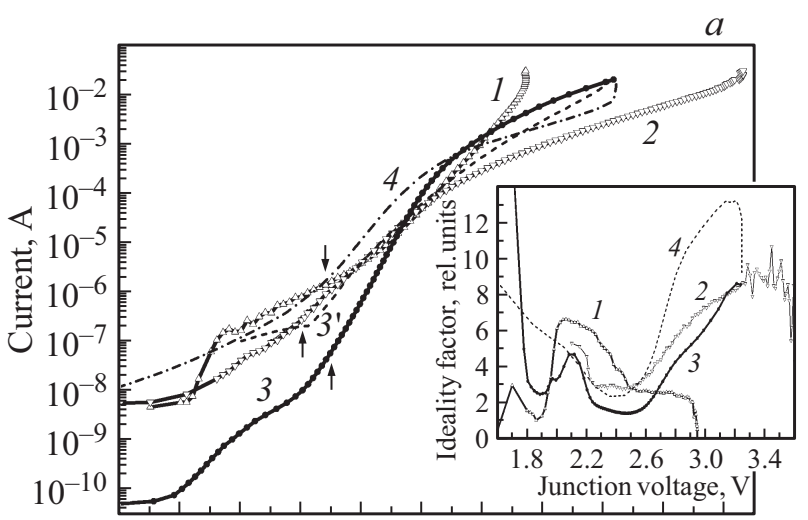

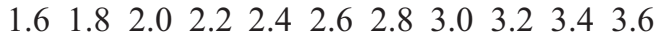
Junction voltage, $\mathrm{V}$
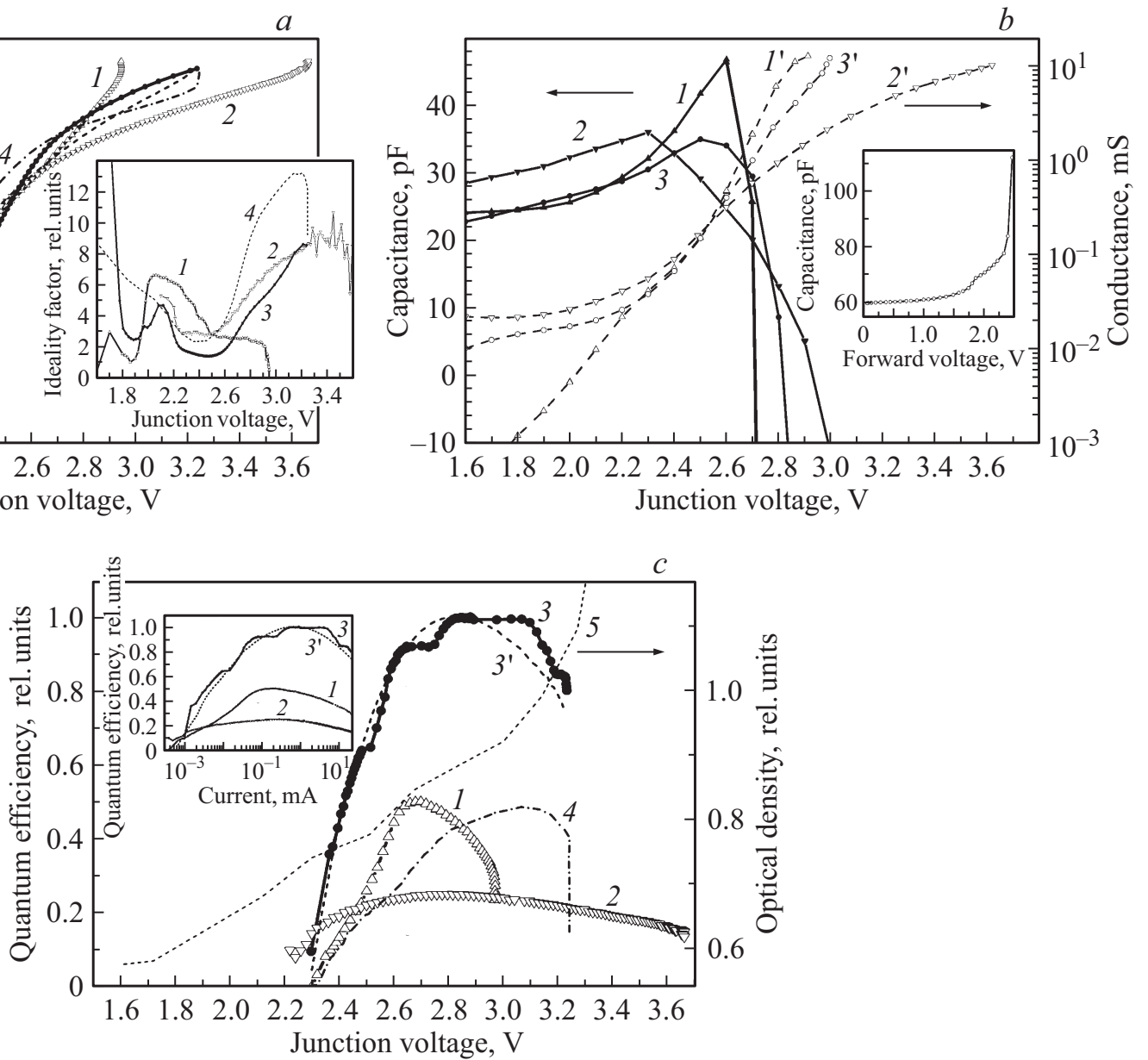

Рис. 1. $a$ - зависимости тока от прямого смещения $p-n$-перехода $V_{j}=V-I r_{s}$ для GaN-светодиодов А $(1), \mathrm{B}(2), \mathrm{C}(3), \mathrm{C}^{*}\left(3^{\prime}\right)$, $\mathrm{D}(4)$. Стрелками на кривых $1-3$ отмечены пороговые напряжения $V_{\mathrm{th}} . r_{s}$, Ом: $1-11.5,2-20,3-21,3^{\prime}-16.5,4-66$. На вставке - зависимости фактора идеальности $I\left(V_{j}\right)$-характеристик от прямого смещения $V_{j}$ для светодиодов А $(1)$, В $(2), \mathrm{C}(3)$ и $\mathrm{D}(4) . b-$ зависимости емкости $(1-3)$ и активной проводимости $\left(1^{\prime}-3^{\prime}\right)$ на частоте 1 МГц от прямого смещения $p-n$-перехода для светодиодов А $\left(1,1^{\prime}\right)$, В $\left(2,2^{\prime}\right)$, С $\left(3,3^{\prime}\right)$. На вставке - зависимость емкости на частоте 100 кГц от прямого смещения $p-n$-перехода для светодиода С. $c$ - зависимости квантовой эффективности электролюминесценции от прямого смещения $V_{j}$ для светодиодов А (1), В (2), C (3), C $\mathrm{C}^{*}\left(3^{\prime}\right), \mathrm{D}(4)$ и зависимость огибающей спектра оптической плотности от энергии фотона для эпитаксиального слоя $n$-GaN. На вставке - токовые зависимости квантовой эффективности для светодиодов А (1), В (2), С (3) и $\mathrm{C}^{*}\left(3^{\prime}\right)$.

$h v_{p}=2.7$ эВ (синие диоды C). Рабочий ток диодов $20 \mathrm{мA}$, реальная площадь $\sim 10^{-3} \mathrm{~cm}^{2}$. Проведены также исследования диодов С после деградации оптической мощности при рабочем токе на 7\% (диоды $\mathrm{C}^{*}$ ) и вертикальных зеленых диодов D c множественными квантовыми ямами $\left(h v_{p}=2.34\right.$ эВ).

Измерения вольт-амперных и вольт-фарадных характеристик проведены с помощью Keithley 238 и CV анализатора Keithley 590, измерения интенсивности излучения проведены с помощью интегрирующей сферы и калиброванного Si-фотодиода. Измерения спектров фотолюминесценции эпитаксиальных слоев $n-\mathrm{GaN}$ проводились при комнатной температуре с помощью спектрометра Avantes. Фотолюминесценция возбуждалась $\mathrm{He}-\mathrm{Cd}$ лазером на длине волны $\lambda=325 \mathrm{Hм}$ со стороны поверхности роста и детектировалась „на про- свет“ со стороны подложки. Интенсивность возбуждения составляла $1 \mathrm{BT} / \mathrm{cm}^{2}$. Измерения спектров оптической плотности проведены методом оптического пропускания с использованием в качестве источника излучения вольфрамовой лампы накаливания (ОИ-24). Спектры падающего на кристалл и прошедшего через кристалл излучения измерялись с помощью спектрометра Avantes.

\section{3. Экспериментальные результаты}

На рис. 1, $a$ представлены зависимости тока от прямого напряжения на ОПЗ, определенного как $V_{j}=V-I \cdot r_{s}$, для светодиодов А, В и С (кривые $\left.1-3\right)$ $\left(V\right.$ - приложенное к диоду напряжение, $r_{s}-$ последовательное сопротивление, полученное из наклона 
$I-V$-характеристики при рабочем токе). На вставке к рис. 1 представлены зависимости фактора идеальности $I\left(V_{j}\right)$-характеристик $n_{j}=(q / k T) d V_{j} / d \ln I$ от смещения $V_{j}$. Как следует из кривой $n_{j}\left(V_{j}\right)$ для синего диода $\mathrm{C}$ (кривая 3), крутизна $I\left(V_{j}\right)$-характеристики резко изменяется в области смещений, равных в энергетических единицах $q V_{j}=1.85-2.2,2.2-2.5,2.5-2.85,2.85-3.2$ эВ. На наиболее крутом участке ВАХ в области 2.2-2.6 В фактор идеальности уменьшается до $n_{j}<2$. В зеленых диодах А и В (кривые 1,2) при этих смещениях также наблюдается изменение крутизны, но в области 2.2-2.6 B $n_{j}>2$.

Представленные на рис. $1, b$ зависимости дифференциальной емкости $C\left(V_{j}\right)$ (кривые 1-3) и активной проводимости $G\left(V_{j}\right)$ (кривые $I^{\prime}-3^{\prime}$ ) на частоте $1 \mathrm{MГц}$ диодов А, В и С обнаруживают корреляцию с крутизной $I\left(V_{j}\right)$-кривых. С увеличением прямого смещения емкость у всех диодов сначала растет, а затем в области $V_{j}=2.4-2.6 \mathrm{~B}$, на наиболее крутом участке $\mathrm{BAX}$, начинает уменьшаться. При этом активная проводимость быстро растет с напряжением. Емкость, достигая нулевой величины, становится отрицательной, отражая инерционное возрастание проводимости [21], в области напряжений $V_{j}>2.6-2.8 \mathrm{~B}$, когда после уменьшения крутизны BAX (диоды В и С) или замедления ее роста (диод А) вновь начинается рост крутизны $I\left(V_{j}\right)$-кривых. При измерениях на частоте 100 кГц емкость у всех диодов резко растет вблизи $V_{j} \approx 2.5 \mathrm{~B}$, как это иллюстрирует представленная на вставке к рис. $1, b$ $C\left(V_{j}\right)$-характеристика диода С.

Как видно из представленных на рис. $1, c$ зависимостей квантовой эффективности излучения $\eta\left(V_{j}\right)$, у диодов А, В и С наблюдается немонотонное изменение эффективности при увеличении смещения (кривые $1-3$ ). $\mathrm{У}$ диодов С и А наблюдается ступенчатый рост эффективности в областях тех же смещений $q V_{j}=2.2-2.5$, 2.5-2.7 эВ (рис. $1, c$, кривые 3,1 ), что и ступенчатый рост тока (рис. $1, a$, кривые 3,1 ). При дальнейшем росте смещения у диода $\mathrm{C}$ после небольшой ступени роста в области $q V_{j}=2.7-2.8$ эВ и участка с пиковой эффективностью $\eta_{p}$, принятой за единицу, наблюдается ступенчатое уменьшение эффективности, достигающее 20\% при рабочем напряжении. У диода А $\left(h v_{p}=2.46\right.$ эВ $)$ $\eta_{p}=0.5$ при пиковом напряжении $V_{p}=2.7$ эВ и падает на $55 \%$ при рабочем токе $I=20 \mathrm{MA}$. У диода В $\left(h v_{p}=2.34\right.$ эВ) $\eta_{p}=0.25$ при $V_{p}=2.8$ эВ и падает на $44 \%$ при $I=20$ мА. Токовые зависимости эффективности диодов А, В и С представлены на вставке к рис. 1, (кривые 1-3). Таким образом, светодиоды А, В и С подтверждают известный из литературы тренд: чем больше излучаемая длина волны, тем меньше эффективность светодиода и сильнее ее падение с ростом тока $[1,2]$. Отметим, что у диодов А, В и С эффективность достигает пиковой величины при том же смещении $V_{j}$, при котором наблюдается нулевая емкость.

На рис. 2 представлены спектр фотолюминесценции и огибающая спектра оптической плотности $D(h v)$

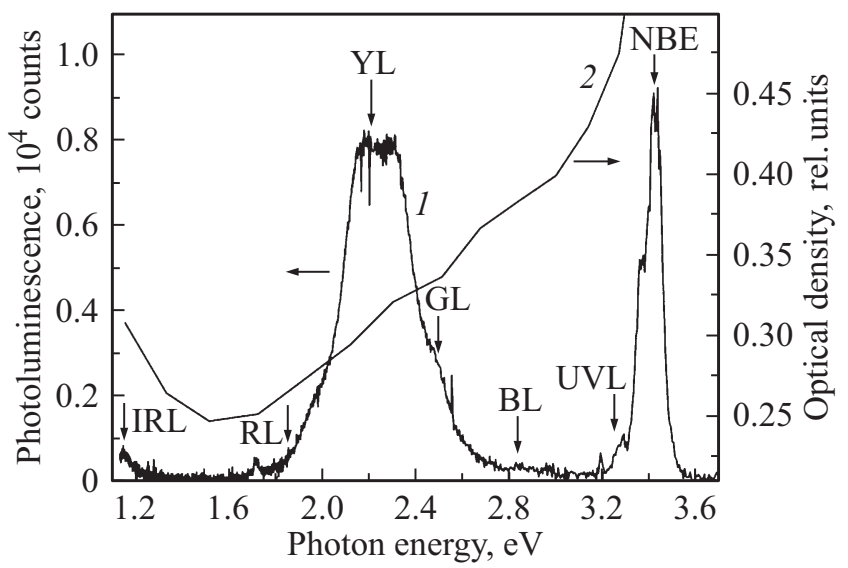

Рис. 2. Спектры фотолюминесценции, возбуждаемой $\mathrm{He}-\mathrm{Cd}-$ лазером $(\lambda=325 \mathrm{Hм})(1)$ и огибающей спектра оптической плотности слоя $\mathrm{GaN}$, выращенного методом MOCVD (2), при $T=300 \mathrm{~K}$. Стрелками отмечены спектральные положения максимумов интенсивности основных полос фотолюминесценции в $\mathrm{GaN}[17]$.

выращенного методом MOCVD эпитаксиального слоя $\mathrm{GaN}$, демонстрирующая ступенчатый рост оптической плотности в области пиковых энергий полос красной $h v_{\mathrm{RL}}=1.85$ эВ, желтой $h v_{\mathrm{YL}}=2.2$ эВ, зеленой $h v_{\mathrm{GL}}=2.5$ эВ, голубой $h v_{\mathrm{BL}}=2.85$ эВ, ультрафиолетовой $h v_{\mathrm{UVL}}=3.2$ эВ и близкраевой фотолюминесценции $h v_{\mathrm{NBE}}=3.4$ эВ $[17,18]$. Сравнение зависимостей $I\left(q V_{j}\right)$, $C\left(q V_{j}\right), G\left(q V_{j}\right)$ и $D(h v)$ позволяет сделать вывод, что ступенчатый рост тока и емкости с ростом прямого смещения и сканирования состояний на границе с квантовой ямой квазиуровнями Ферми коррелирует со ступенчатым ростом плотности глубоких центров $N_{\mathrm{RL}}(E), N_{\mathrm{YL}}(E), N_{\mathrm{GL}}(E), N_{\mathrm{BL}}(E)$ и $N_{\mathrm{UVL}}(E)$ в запрещенной зоне, ответственных за полосы дефектной фотолюминесценции.

\section{4. Обсуждение результатов}

\section{1. Снижение эффективного барьера за счет прыжкового транспорта в области пространственного заряда}

Туннельная прозрачность барьеров в $p$-n-структуре с квантовой ямой $\mathrm{InGaN} / \mathrm{GaN}$ в значительной степени определяется уровнем легирования барьерных слоев. В то же время присутствие глубоких центров облегчает туннелирование носителей через ОПЗ. Спектр излучения формируется в результате излучательной рекомбинации в состояниях хвостов квантовой ямы $\mathrm{InGaN} / \mathrm{GaN}$ [22] и детектируется уже вблизи порогового напряжения. Так, в синем диоде С при $V_{j}=2.4 \mathrm{~B}$ и токе $I=1$ мкА детектируется спектр излучения в широкой области энергий фотонов $h v=2.4-2.8$ эВ с пиковой энергией $h v_{p}=2.6$ эВ. Это свидетельствует о том, что основной вклад в излучение определяется 


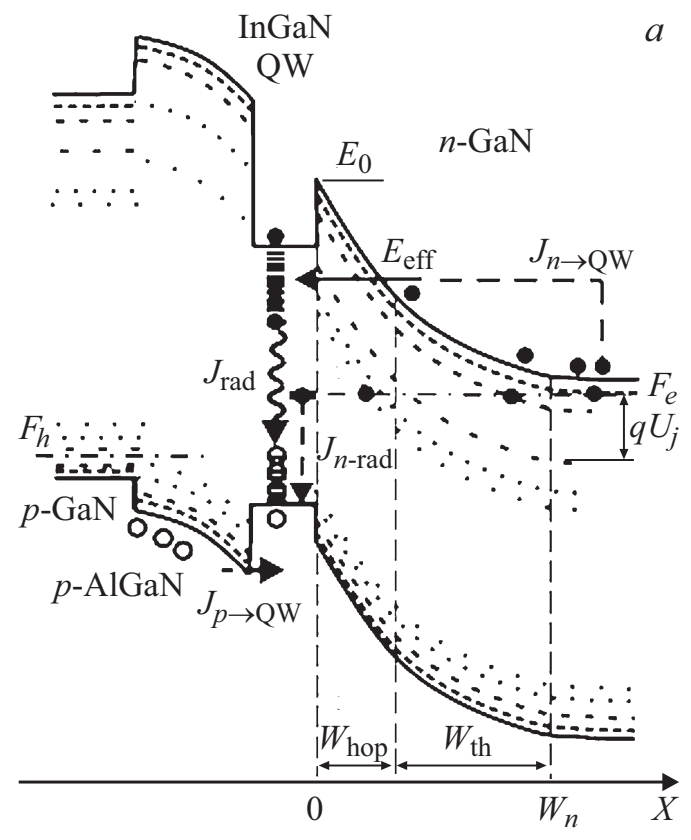

$a$
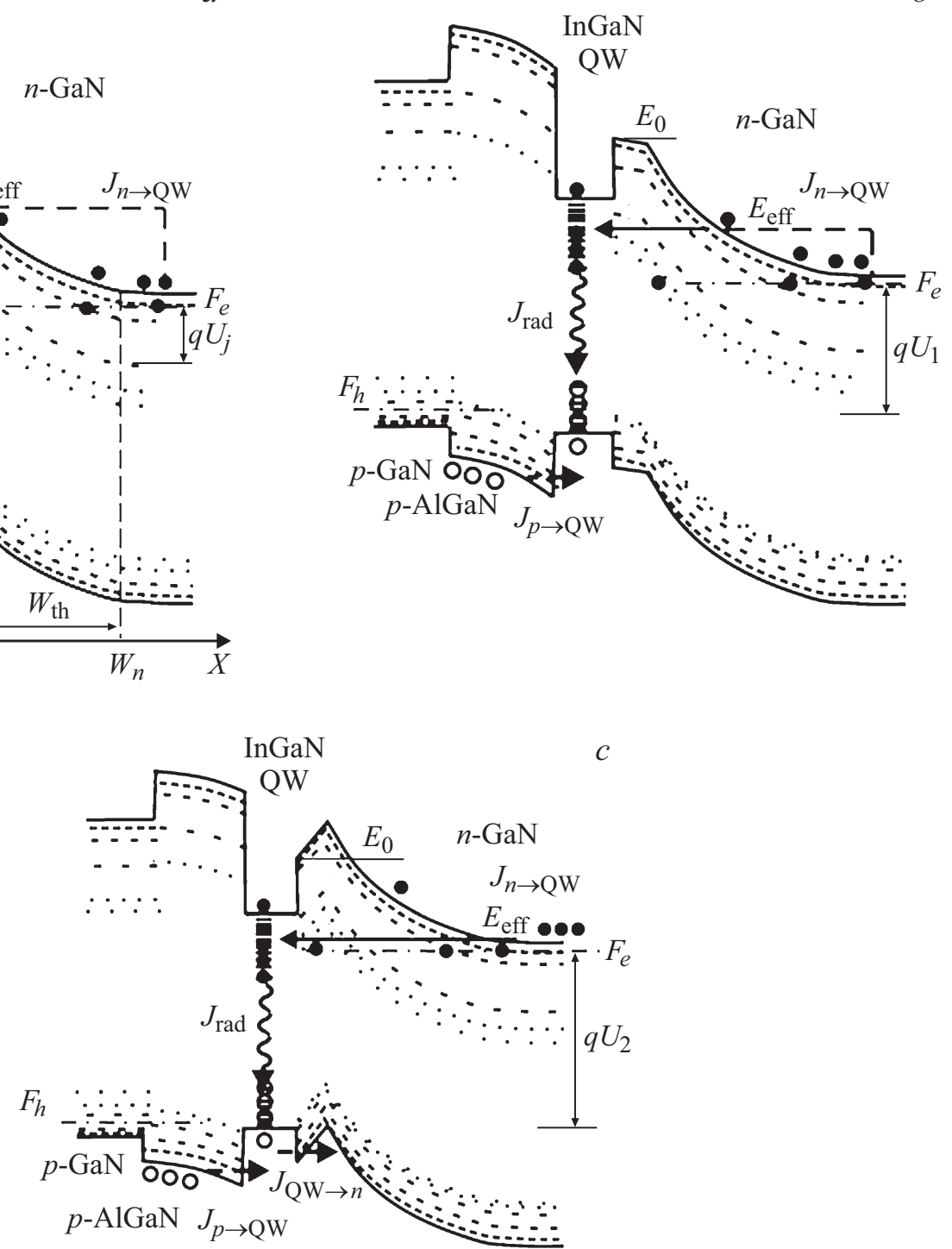

Рис. 3. Схематическое изображение зонной структуры GaN-светодиода с одиночной квантовой ямой $\mathrm{InGaN} / \mathrm{GaN}$ при прямом смещении. Пунктирными линиями отмечены максимумы плотности состояний для центров окраски, ответственных за полосы дефектной фотолюминесценции. $a-q V_{j} \approx q V_{\mathrm{th}}, b-q V_{1}<q V_{p}, c-q V_{2}>q V_{p}$. $J_{n \rightarrow \mathrm{QW}}$ и $J_{p \rightarrow \mathrm{QW}}$ - электронная и дырочная компоненты туннельного тока с участием глубоких центров через ОПЗ, $E_{\mathrm{eff}}$ - транспортная энергия для электронов, формирующих пик в спектре излучения.

туннелированием через барьеры термоактивированных носителей [18]. В диодах А, В и С на границе с квантовой ямой имеется слабо легированная область $n$-GaN $[20,23]$, так что полный ток в барьерной структуре этих диодов контролируется электронным туннельным током. Для иллюстрации механизма туннелирования с участием дефектов (defect-assisted tunneling, hopping) будем обращаться к электронному току (рис. 3, $a$ ).

Прыжковое сопротивление при упругом рассеянии зависит от плотности состояний на транспортном уровне $E_{t e}$. Локальная прыжковая проводимость в ОПЗ может быть записана в виде: $\sigma_{\text {hop }}=q^{2} g\left(E_{\text {loc }}\right) R_{i j}^{2} v_{\text {hop }}$, где $g\left(E_{\mathrm{loc}}\right)$ - распределение состояний по энергии локали- зации $E_{\mathrm{loc}}(x)=E_{c}-E(x)$ глубоких центров, $R_{i j}-$ расстояние между центрами, $v_{\text {hop }}\left(R_{i j}\right)=v_{0} \exp \left(-2 \gamma R_{i j}\right)-$ частота прыжков, $v_{0} \approx 10^{12} \mathrm{c}^{-1}$ - частота попыток, $\gamma=1 / a-$ степень перекрытия волновых функций, $a-$ боровский радиус [24].

Вблизи дна зоны проводимости $\mathrm{GaN}$ энергетическое распределение плотности состояний может быть аппроксимировано экспоненциальной функцией $g\left(E_{\mathrm{loc}}\right)=\left(N_{\mathrm{L}} / E_{U}\right) \exp \left(-E_{\mathrm{loc}} / E_{U}\right)$ с урбаховской энергией $E_{U}=0.05$ эВ [11], где $N_{\mathrm{L}}-$ полное число состояний. Вероятность прыжка по мере приближения к квантовой яме экспоненциально уменьшается из-за уменьшения плотности состояний $g\left(E_{\mathrm{loc}}\right)$. Распределенное прыжковое 
сопротивление ОПЗ определяется локальным прыжковым сопротивлением вблизи квантовой ямы $r_{\text {hop }}\left(E_{\mathrm{te}}\right.$, $0)$. С увеличением транспортной энергии $E_{\text {te }}$ частота прыжков на границе с квантовой ямой экспоненциально растет с ростом плотности состояний, которая в свою очередь экспоненциально увеличивается с уменьшением энергии локализации $E_{\mathrm{loc}}(0)$ :

$$
v_{\text {hop }}\left(E_{\text {loc }}\right)=v_{0} \exp \left[-2 \gamma N_{\mathrm{L}}^{-1 / 3} \exp \left(E_{\mathrm{loc}} / 3 E_{U}\right)\right] .
$$

Согласно оценкам, сделанным в [19] в предположении $N_{\mathrm{L}}=N_{c}=2.3 \cdot 10^{18} \mathrm{~cm}^{-3}[25]\left(N_{c}-\right.$ эффективная плотность состояний в зоне проводимости $\mathrm{GaN}$ ), для $E_{\mathrm{te}} \geq E_{c}-0.15$ эВ частота перескоков у квантовой ямы увеличивается с ростом $E_{\mathrm{te}}$ от $10^{5}$ до $10^{10} \mathrm{c}^{-1}$, а локальная проводимость от $10^{-7}$ до $10^{-2} \mathrm{OM}^{-1} \cdot \mathrm{cm}^{-1}$.

Для более глубоких транспортных уровней $E_{\mathrm{te}} \leq E_{c}-0.15$ эВ прыжковое сопротивление $r_{\mathrm{hop}}\left(E_{\mathrm{te}}, 0\right)$ определяется энергетическим распределением глубоких центров. Так как полосы желтой, голубой и зеленой фотолюминесценции в $\mathrm{GaN}$ имеют гауссову форму [17], энергетическое распределение глубоких центров, ответственных за полосы оптического поглощения, может быть также представлено в виде функций Гаусса:

$$
g\left(E_{\mathrm{loc}}\right)=\frac{2 N_{\mathrm{L}}}{\sigma \sqrt{\pi}} \exp \left(-\frac{\left(E_{\mathrm{loc}}-E_{0}\right)^{2}}{2 \sigma^{2}}\right),
$$

где $\sigma=w_{\mathrm{L}} /(2 \sqrt{\ln 4})-$ среднеквадратичное отклонение, $w_{\mathrm{L}}$ - полная ширина на половине высоты полосы фотолюминесценции (FWHM), для $N_{\mathrm{YL}}(E), N_{\mathrm{GL}}(E)$ равная $w_{\mathrm{L}} \approx 400$ мэВ [17], $E_{0}-$ положение максимума гауссиана, соответствующее энергии фотона в максимуме оптического поглощения.

При увеличении прямого смещения от порогового, равного $q V_{\text {th }} \approx 2.2-2.3$ эВ, до $q V_{j} \approx 2.5$ эВ квазиуровень Ферми на границе с квантовой ямой $\mathrm{F}_{e}(0)$ пересекает полосу глубоких центров $N_{\mathrm{YL}}(E, 0)$. При $E_{0} / 2>\mathrm{F}_{e}(0)>q V_{\mathrm{th}}$ плотность состояний $N_{\mathrm{YL}}\left(\mathrm{F}_{e}, 0\right)$ экспоненциально растет, затем рост $N_{\mathrm{YL}}\left(\mathrm{F}_{e}, 0\right)$ замедляется, достигая максимального значения. Прыжковая проводимость на квазиуровне Ферми $\sigma_{\mathrm{hop}}\left(\mathrm{F}_{e}, 0\right)$ экспоненциально увеличивается с ростом $N_{\mathrm{YL}}(E, 0)$ : $v_{\text {hop }} \sim \exp \left[-2 \gamma\left(g\left(E_{\text {loc }}\right)^{-1 / 3} k T\right)\right]$. Так, согласно оценкам [18], при $N_{\mathrm{YL}}=10^{17} \mathrm{~cm}^{-3}$ проводимость достигает величины $\sigma_{\text {hop }}=2 \cdot 10^{-7} \mathrm{OM}^{-1} \mathrm{~cm}^{-1}$ и увеличивается на порядок при увеличении плотности состояний вдвое, характеристическая энергия на краю полосы составляет $\sim k T$, возрастая до $\sim 2 k T$ на половине высоты пика полосы распределения [18]. Спад плотности $N_{\mathrm{YL}}(E, 0)$ на высокоэнергетичном краю распределения компенсируется уменьшением ширины области, занимаемой этими центрами на квазиуровне Ферми.

Дифференциальное сопротивление $n$-барьера $r_{n}$ для потока термоактивированных электронов с энергией $E_{\mathrm{te}}$ из нейтральной $n$-области в изоэнергетические состояния квантовой ямы равно сумме дифференциального сопротивления эффективного $n$-барьера $r_{j}$ высотой
$E_{\mathrm{te}}-\mathrm{F}_{e}$ и шириной $w_{\text {th }}$ и прыжкового сопротивления области туннелирования $r_{\text {hop }}$ шириной $w_{\text {hop }}, r_{n}=r_{j}+r_{\text {hop }}$ (рис. $3, a)$. В части ОПЗ шириной $w_{\mathrm{th}}\left(E_{\mathrm{te}}\right)$ термоактивированные электроны свободны и движутся с тепловой скоростью. Так как электроны имеют больцмановское распределение по энергии, то для транспортной энергии $E_{\mathrm{te}}$ эквивалентное дифференциальное сопротивление $r_{j}\left(E_{\mathrm{te}}\right)$, экспоненциально увеличивающееся с увеличением энергии термоактивированных электронов $E_{\mathrm{te}}$, с ростом смещения $q V_{j}=\mathrm{F}_{e}$ экспоненциально уменьшается: $\left.r_{j}\left(E_{\mathrm{te}}\right) \sim \exp \left(\left(E_{\mathrm{te}}-\mathrm{F}_{e}\right) / k T\right)\right)$.

Сквозной электронный ток ограничивается распределенным прыжковым сопротивлением области туннелирования, определяющимся высоким локальным сопротивлением вблизи квантовой ямы, $r_{\mathrm{hop}}\left(E_{\mathrm{te}}, 0\right)$. Основной поток электронов протекает на квазиуровне Ферми и ограничен прыжковым сопротивлением $r_{\mathrm{hop}}\left(\mathrm{F}_{e}, 0\right)$. При $V_{j}=V_{\text {th }}$ приложенное внешнее напряжение падает преимущественно вблизи квантовой ямы, спрямляя изгиб зон, так что треугольный $n$-барьер у границы приближается к прямоугольному (рис. $3, b)$. При $V_{j}>V_{\text {th }}$ сопротивление $r_{j}\left(E_{\mathrm{te}}\right)$ экспоненциально уменьшается с увеличением смещения, сопротивление $r_{\text {hop }}\left(\mathrm{F}_{e}, 0\right)$ также уменьшается, так как увеличивается плотность локализованных состояний на границе с квантовой ямой $N_{\mathrm{YL}}$ $(\mathrm{F}, 0)$, пересекаемых квазиуровнем Ферми $\mathrm{F}_{e}$. Это приводит к ступенчатому увеличению тока в области $2.2-2.5$ В (рис. $1, a$ ). При дальнейшем увеличении прямого смещения широкие перекрывающиеся гауссовы полосы центров окраски $N_{\mathrm{GL}}(E), N_{\mathrm{BL}}(E)$ и $N_{\mathrm{UVL}}(E)$ приводят к уменьшению $r_{\mathrm{hop}}\left(\mathrm{F}_{e}, 0\right)$ в области максимумов плотности отдельных полос, что приводит к ступенчатому росту тока.

Отметим, что в рамках этой модели последовательное сопротивление $r_{s}$, определяемое из наклона $I(V)$-характеристики при рабочем напряжении (разд. 3), является суммой прыжкового сопротивления ОПЗ $r_{\text {hop }}$ и последовательного сопротивления диода (сопротивления базы и контактов) $R_{s}: r_{s}=R_{s}+r_{\text {hop, }}$, чо может обусловливать близкие к вертикальным участки на $I\left(V_{j}\right)$-характеристиках в области рабочих токов (рис. $1, a)$.

Таким образом, ступенчатый рост тока в прямосмещенном светодиоде, наблюдающийся когда разность квазиуровней Ферми достигает величины оптических пороговых энергий в спектрах поглощения, можно связать с пересечением квазиуровнями Ферми на границе с квантовой ямой полос локализованных состояний, ответственных за полосы дефектной фотолюминесценции в GaN.

\section{2. Распределение электрического поля в ОПЗ с прыжковой проводимостью}

Прыжковая проводимость по глубоким центрам в ОПЗ приводит к кардинальному изменению переходных процессов в диоде. При быстром включении прямого напряжения переходной процесс в ОПЗ определяется 
распределением локальных времен диэлектрической релаксации $\tau_{r}\left(E_{\mathrm{loc}}, x\right)=\varepsilon_{0} \varepsilon r_{\mathrm{hop}}\left(E_{\mathrm{loc}}, x\right) \quad\left(\varepsilon_{0}-\right.$ электрическая постоянная, $\varepsilon-$ диэлектрическая проницаемость). При небольших смещениях $V_{j}<\sim 2.5 \mathrm{~B}$ из-за увеличения времени перескока по мере углубления электрона в ОПЗ начинает преобладать захват электронов, и временной масштаб $\tau_{r}(E, x)$ увеличивается от наносекундного до миллисекундного [19]. Переходной ток затухает со временем как $I(t) \sim 1 / t[19]$, что приводит к емкостному характеру токового отклика диода. На переменном напряжении, повышение частоты уменьшает емкостной отклик [19]. Увеличение прямого смещения приводит к заполнению глубоких центров в полосе энергий, пересекаемых перемещающимся с ростом $V_{j}$ вверх квазиуровнем Ферми $\mathrm{F}_{e}$. В результате плотность состояний на $\mathrm{F}_{e}$ после перемещения увеличивается, инерционное возрастание проводимости проявляется как индуктивный токовый отклик диода (отрицательная емкость) [23], доминирующий при смещениях $V_{j}>\sim 2.5 \mathrm{~B}$.

Описанный процесс формирования емкостного/индуктивного токового отклика диода с прыжковой проводимостью в ОПЗ позволяет дать объяснение отмеченной в разд. 3 корреляции ступенчатого хода кривых $C\left(V_{j}\right)$, $G\left(V_{j}\right)$ и $I\left(V_{j}\right)$ (рис. $\left.1, a, b\right)$.

Так, для синего диода С при $V_{j}=0$ величина емкости на частоте $1 \mathrm{MГц,} C_{0}=19 п \Phi$, втрое меньше, чем емкость на частоте 100 кГц, $C_{0}=60$ пФ (см. вставку на рис. $1, b)$. Ступенчатый рост емкости $(f=100$ кГц $)$ в области $q V_{j}=1.85-2.2$ и 2.2-2.5 эВ коррелирует со ступенчатым ростом тока (рис. $1, a$, кривая 3 ) и связан с пересечением квазиуровнями Ферми гауссовых полос состояний с плотностью $N_{\mathrm{RL}}(E)$ и $N_{\mathrm{YL}}(E)$. При $V_{j}>2.45 \mathrm{~B}$ сначала наблюдается уменьшение емкости до нулевого значения, а затем отрицательная емкость, величина которой достигает $C=-1600 п \Phi$ при $V_{j}=3.2 \mathrm{~B}$. При этом реактивное сопротивление уменьшается до $-1 / \omega C=100$ Ом, но полная проводимость на частоте 1 МГц остается меньше проводимости на постоянном токе $G_{d c}$. На частоте 100 кГц при $V_{j}>2.45 \mathrm{~B} G \approx G_{d c}$, a емкость демонстрирует почти вертикальный рост, подобно тому как растет барьерная емкость $C_{b}$ диода на основе материала, с меньшей почти на 1 эВ шириной запрещенной зоны, чем у $\mathrm{GaN}$ (и $\left.\sigma_{\mathrm{hop}}=0\right)$, при приближении смещения к диффузионному потенциалу, равному $V_{b i}=2.45 \mathrm{~B}: C_{b} \sim\left(V_{b i}-V\right)^{-1 / 2}$. Подобный характер имеют и зависимости $C\left(V_{j}\right)$ зеленых диодов А и В.

Результаты емкостных измерений позволяют сделать вывод о том, что в процессе диэлектрической релаксации в ОПЗ с прыжковой проводимостью устанавливается стационарное распределение потенциала, определяющееся распределенным сопротивлением $r_{\text {hop }}\left(\mathrm{F}_{e}, x\right)$. При небольших прямых смещениях, когда на переменном напряжении доминирует емкостной токовый отклик диода и $\omega C r_{\text {hop }}>1$, это приводит к уменьшению поля вблизи квантовой ямы (спрямлению зон); при больших смещениях, когда доминируют индуктивный $(f=1$ МГц $)$ и активный $(f=100$ кГц) токовые отклики и $\omega C r_{\text {hop }}<1,-$ к изменению направления поля у квантовой ямы (изгибу зон вверх).

\section{3. Влияние прыжковой проводимости в области пространственного заряда на квантовую эффективность зеленых и синих светодиодов}

4.3.1. Рост эффективности при низких уровнях инжекции. Как показало сравнение экспериментальных зависимостей тока $I\left(V_{j}\right)$ (рис. $1, a$, кривая 3 ), интенсивности $I_{\mathrm{EL}}\left(V_{j}\right)$ и эффективности излучения $\eta\left(V_{j}\right) \sim I_{\mathrm{EL}} / I$ (рис. 1, , кривая 3) в синем диоде C, основной вклад в ступенчатый характер роста эффективности вносит быстрый рост интенсивности излучения. Так, вблизи порогового напряжения $I_{\mathrm{EL}}$ быстро растет, увеличиваясь на порядок, как $I_{\mathrm{EL}} \sim \exp \left(q V_{j} / n k T\right)$, где $n \approx 1$.

Анализ ступенчатого хода кривых $\eta\left(q V_{j}\right)$ с ростом смещения позволяет предложить следующий механизм влияния глубоких центров в $\mathrm{GaN}$ на эффективность светодиодов.

Ограничение потока термоактивированных электронов с энергией $E_{\mathrm{te}}>\mathrm{F}_{e}$ в квантовую яму увеличивается с увеличением отношения $r_{\mathrm{hop}}\left(\mathrm{F}_{e}\right) / r_{j}\left(E_{\mathrm{te}}\right)$ (разд. 4.1). При увеличении смещения сопротивление $r_{j}\left(E_{\mathrm{te}}\right)$ экспоненциально уменьшается с энергией активации $k T$. В то же время прыжковое сопротивление $r_{\text {hop }}\left(\mathrm{F}_{e}\right)$ при пересечении квазиуровнем Ферми у квантовой ямы гауссовых полос дефектных центров меняется немонотонно: быстро уменьшается, когда у квантовой ямы $\mathrm{F}_{e}(0)$ достигает оптической пороговой энергии, относительно слабо меняется в области ширины на половине высоты и вновь быстро уменьшается на высокоэнергетичном краю полосы. При быстром уменьшении $r_{\mathrm{hop}}\left(\mathrm{F}_{e}\right)$ c ростом $q V_{j}$ поток термоактивированных электронов слабее ограничивается прыжковым сопротивлением, и эффективность растет. Рост эффективности прекращается, когда отношение $r_{\mathrm{hop}}\left(\mathrm{F}_{e}\right) / r_{j}\left(E_{\mathrm{te}}\right)$ начинает расти. Падение напряжения на $r_{\text {hop }}\left(\mathrm{F}_{e}\right)$ приводит к выпрямлению зон вблизи квантовой ямы (рис. 3,b). Так как дефекты образуют хвост акцепторных состояний у валентной зоны [17], выпрямление зон приводит к увеличению туннельного потока дырок из квантовой ямы и ограничению эффективности. Ступенчатые токовые зависимости эффективности (см. вставку на рис. 1,c) подтверждают эту модель. Предложенная модель влияния глубоких центров в $\mathrm{GaN}$ на эффективность излучения квантовых ям In GaN/GaN подтверждается также ступенчатым ростом спектральной квантовой эффективности излучения $\eta_{h v}$ с напряжением, который наблюдался нами ранее в [26,27] в диодах С в области тех же смещений (см. рис. 4 в [26]), что и рост квантовой эффективности $\eta$ (рис. 2, с, кривая 3).

4.3.2. Падение эффективности при высоких уровнях инжекции. Как отмечено в разд. 3 , эффективность начинает падать при пиковых напряжениях $V_{p}$, больших, чем напряжение, при котором наблюдается нулевая емкость 
на частоте 1 МГц. Это указывает на связь падения эффективности с резистивным характером токового отклика диода. В свою очередь это позволяет предположить, что на эффективность оказывают влияние изменение направления поля вблизи квантовой ямы и изгиб зон вверх (рис. $3, c)$, подобно полю в $p-i-n$-структуре при больших прямых напряжениях. Падение напряжения на прыжковом сопротивлении $r_{\text {hop }}\left(q V_{j}\right)$ создает поле, превышающее встроенное электростатическое поле, создаваемое контактной разностью потенциалов. Ослабление конфайнмента дырок в квантовой яме InGaN/GaN полем, вытягивающим дырки из квантовой ямы InGaN/GaN, приводит к падению эффективности (рис. 3,c). Отметим, что резкое возрастание плотности состояний на квазиуровне Ферми $\mathrm{F}_{e}(0)$ замедляет падение эффективности из-за уменьшения отношения $r_{\mathrm{hop}}\left(\mathrm{F}_{e}\right) / r_{j}\left(E_{\mathrm{te}}\right)$ и уменьшения падения напряжения вблизи квантовой ямы. Так, на кривой $\eta\left(q V_{j}\right)$ диода $\mathrm{C}$ (рис. $1, c$, кривая 3$)$ при $V_{j}=3.2$ В наблюдается небольшая ступень, вызванная ростом на квазиуровне Ферми $\mathrm{F}_{e}(0)$ плотности $N_{\mathrm{UVL}}(E)$.

В работе [21] рост плотности заполненных локализованных состояний на границе квантовой ямы $\mathrm{InGaN} / \mathrm{GaN}$ с увеличением смещения $q V_{j}$ и соответственно с увеличением разности между квазиуровнями Ферми, проявляющийся в индуктивном токовом отклике $p-n$-структуры, интерпретировался как свидетельствующий о росте числа рекомбинационных центров и уменьшении безызлучательного времени жизни на границе квантовой ямы с ростом уровня инжекции, приводящим к падению эффективности. Возникновение в $n$-барьере у границы с квантовой ямой сильного поля, вытягивающего дырки из квантовой ямы, рассмотренное в данной работе, расширяет область безызлучательной рекомбинации носителей в область $n$-барьера.

Крутизна $I-V_{j}$-характеристик зеленых диодов А и В в области напряжений $V_{j}=2.2-2.6 \mathrm{~B}$ ниже, чем у синего диода С (рис. 1,a). В области напряжений $V_{j}=2.3-2.6 \mathrm{~B}$, когда наблюдается рост эффективности, фактор идеальности $I-V_{j}$-характеристик в зеленых диодах $\mathrm{A}$ и $\mathrm{B} \eta_{j} \geq 3$, тогда как и у синего диода С $\eta_{j} \leq 2$, как в диоде с надбарьерной инжекцией (см. разд. 3). Это указывает на то, что в зеленых диодах протекание термоактивированных электронов сильнее ограничено прыжковым сопротивлением (см. разд. 4.1). Величина тока в области $V_{j}<2.5 \mathrm{~B}$ в зеленых диодах А и В значительно выше, чем в синем диоде С, а при $V_{j}>2.5 \mathrm{~B}$ в диоде В и $V_{j}=2.5-2.8 \mathrm{~B}$ в диоде $\mathrm{A}-$ ниже. Это свидетельствует о большей плотности глубоких состояний $N_{\mathrm{RL}}(E)$ и $N_{\mathrm{YL}}(E)$, ответственных за красную и желтую фотолюминесценцию, и меньшей плотности более мелких состояний $N_{\mathrm{BL}}(E)$ и $N_{\mathrm{UVL}}(E C)$, ответственных за голубую и УФ фотолюминесценцию, в зеленых светодиодах. Соответственно это приводит к большой величине отношения сопротивлений $r_{\mathrm{hop}}\left(\mathrm{F}_{e}\right) / r_{j}\left(E_{\mathrm{te}}\right)$ (см. разд. 4.3.1) при $V_{j}>2.5 \mathrm{~B}$ и низкой эффективности зеленых светодиодов.
Как показала проведенная нами характеризация GaNсветодиодов различных производителей, малая крутизна $I-V_{j}$-характеристик является типичной чертой зеленых светодиодов. В качестве примера на рис. $1, a, c$, а также на вставке к рис. 1, $a$, представлены зависимости $I\left(V_{j}\right), \eta\left(V_{j}\right)$ и $n_{j}\left(V_{j}\right)$ (кривые 4 ) для вертикального зеленого светодиода D с множественными квантовыми ямами $\mathrm{InGaN} / \mathrm{GaN}\left(h v_{p}=2.34\right.$ эB). Малая крутизна $I-V_{j}$-кривых в области $V_{j}>2.5 \mathrm{~B}$ указывает на относительно малую плотность состояний $N_{\mathrm{GL}}(E, 0)$ и $N_{\mathrm{BL}}(E, 0)$, вызывающую большую величину прыжкового сопротивления $r_{\text {hop }}(E, x)$ и низкую эффективность.

Отметим также, что подобная трансформация энергетического спектра дефектов в $\mathrm{GaN}$, проявляющаяся в увеличении тока при малых напряжениях и уменьшении крутизны BAX в GaN-светодиодах, характерна для начальной стадии процесса старения и деградации их оптической мощности (см., например, [28]), что связывается в [29] с влиянием температуры на стабильность водородсодержащих центров окраски $V_{\mathrm{Ga}} \mathrm{H}_{n}-\mathrm{O}_{N}$, ответственных за голубую фотолюминесценцию. Предполагается, что депассивация гидрогенизированных вакансий галлия $V_{\mathrm{Ga}} \mathrm{H}_{n}$ вызывает увеличение концентрации центров окраски $V_{\mathrm{Ga}} \mathrm{O}_{N}$, ответственных за желтую фотолюминесценцию [18]. Так, после деградации оптической мощности при рабочих токах на 7\% у диода $C^{*}$ наблюдается уменьшение крутизны ВАХ и уменьшение эффективности при $V_{j}>2.8 \mathrm{~B}$ (рис. $1, a, c$, кривые $3^{\prime}$ ). Косвенным подтверждением более высокой плотности $N_{\mathrm{YL}}(E)$ в зеленых светодиодах служит также наблюдавшееся в [30] экспоненциальное увеличение концентрации инкорпорируемого кислорода при повышении концентрации индия в MOCVD-слоях InGaN.

Результаты данной работы дают основания связать понижение эффективности квантовых ям InGaN/GaN при уменьшении пиковой энергии излучения с изменением энергетического спектра центров окраски вблизи границ с активной областью и доминированием дефектов, ответственных на желтую фотолюминесценцию.

\section{5. Заключение}

Высокая плотность глубоких центров в $\mathrm{GaN}$ приводит к возникновению прыжковой проводимости через область пространственного заряда в $p-n$-структурах с квантовыми ямами $\mathrm{InGaN} / \mathrm{GaN}$ и обеспечивает инжекцию термоактивированных электронов с энергией выше квазиуровня Ферми в квантовую яму уже при низких пороговых напряжениях $\left(V_{\text {th }}=2.2-2.3 \mathrm{~B}\right)$.

Прыжковая проводимость ОПЗ определяется энергетическим спектром дефектов в $\mathrm{GaN}$ и с увеличением уровня инжекции все больше ограничивает прохождение потока термоактивированных носителей в активную область. Падение напряжения на наиболее резистивной части ОПЗ вблизи квантовой ямы искажает форму 
потенциального барьера, выпрямляя зоны, а с увеличением тока, - изменяя направление электрического поля вблизи границ с квантовой ямой. Это приводит к ослаблению конфайнмента носителей в квантовой яме InGaN/GaN, увеличению туннельного потока дырок из квантовой ямы, их безызлучательной рекомбинации в области $n$-барьера и падению эффективности.

Низкая прыжковая проводимость ОПЗ в зеленых $\mathrm{GaN}$ светодиодах, приводящая к низкой эффективности, связана с доминированием глубоких центров в энергетическом спектре дефектов и недостаточной плотностью мелких центров.

\section{Список литературы}

[1] M. Peter, A. Laubsch, P. Stauss, A. Walter, J. Baur, B. Hahn. Phys. Status Solidi C, 5 (6), 2050 (2008).

[2] D. Schiavon, M. Binder, M. Peter, B. Galler, P. Drechsel, F. Scholz. Phys. Status Solidi B, 250 (2), 283 (2013).

[3] А.Ф. Цацульников, В.В. Лундин, А.В. Сахаров, Е.Е. Заварин, С.О. Усов, А.Е. Николаев, Н.В. Крыжановская, М.А. Синицын, В.С. Сизов, А.Л. Закгейм, М.Н. Мизеров. ФТП, 44 (6), 837 (2010).

[4] T. Wang. Semicond. Sci. Technol., 31, 093003 (2016).

[5] M.A. Maur, A. Pecchia, G. Penazzi, W. Rodrigues, A.D. Carlo. Phys. Rev. Lett., 116, 027401 (2016).

[6] S. Hammersley, M.J. Kappers, F.C.-P. Massabuau, S.L. Sahonta, P. Dawson, R.A. Oliver, C.J. Humphreys. Appl. Phys. Lett., 107, 132106 (2015).

[7] P. Perlin, M. Osinski, P.G. Eliseev, V.A. Smagley, J. Mu, M. Banas, P. Sartori. Appl. Phys. Lett., 69, 1680 (1996).

[8] В.Е. Кудряшов, А.Н. Туркин, А.Э. Юнович, А.Н. Ковалев, Ф.И. Маняхин. ФТП, 33, 445 (1999).

[9] Н.И. Бочкарева, Е.А. Zhirnov, А.А. Ефремов, Ю.Т. Ребане, Р.И. Горбунов, Ю.Г. Шретер. ФТП, 39, 627 (2005).

[10] M. Mandurrino, G. Verzellesi, M. Goano, M. Vallone, F. Bertazzi, G. Ghione, M. Meneghini, G. Meneghesso, E. Zanoni. Phys. Status Solidi A, 212, 947 (2015).

[11] C.H. Qiu, C. Hoggatt, W. Melton, M.W. Leksono, J.I. Pankove. Appl. Phys. Lett., 66, 2712 (1995).

[12] A. Hierro, D. Kwon, S.A. Ringel, M. Hansen, J.S. Speck, U.K. Mishra, S.P. DenBaars. Appl. Phys. Lett., 76, 3064 (2000).

[13] O. Ambacher, W. Reiger, P. Ansmann, H. Angerer, T.D. Moustakas, M. Stutzmann. Sol. St. Commun., 97, 365 (1996).

[14] P.B. Klein, S.C. Binari. J. Phys.: Condens. Matter, 15, R1641 (2003).

[15] L. Balagurov, P.J. Chong. Appl. Phys. Lett., 68, 43 (1996).

[16] S. Pimputkar, S. Suihkonen, M. Imade, Y. Mori, J.S. Speck, S. Nakamura. J. Cryst. Growth, 432, 49 (2015).

[17] . M.A. Reshchikov, H. Morkoç. J. Appl. Phys., 97, 061301 (2005).

[18] Н.И. Бочкарева, И.А. Шеремет, Ю.Г. Шретер. ФТП, 50 (10), 1387 (2016).

[19] Н.И. Бочкарева, В.В. Вороненков, Р.И. Горбунов, М.В. Вирко, В.С. Коготков, А.А. Леонидов, П.Н. ВоронцовВельяминов, И.А. Шеремет, Ю.Г. Шретер. ФТП, 51 (9), 1235 (2017).
[20] T. Mukai, K. Takekava, S. Nakamura. Jpn. J. Appl. Phys., 37, L839 (1996).

[21] Н.И. Бочкарева, Ю.Т. Ребане, Ю.Г. Шретер. ФТП, 49 (12), 1714 (2015).

[22] S. Chichibu, T. Azuhata, T. Sota, S. Nakamura. Appl. Phys. Lett., 69, 4188 (1996).

[23] Н.И. Бочкарева, В.В. Вороненков, Р.И. Горбунов, Ф.Е. Латышев, Ю.С. Леликов, Ю.Т. Ребане, А.И. Цюк, Ю.Г. Шретер. ФТП, 47 (1), 129 (2013).

[24] D. Monroe. Phys. Rev. Lett., 54, 146 (1985).

[25] T. Tiedje, A. Rose. Sol. St. Commun., 37, 49 (1980).

[26] N.I. Bochkareva, V.V. Voronenkov, R.I. Gorbunov, A.S. Zubrilov, Y.S. Lelikov, P.E. Latyshev, Y.T. Rebane, A.I. Tsyuk, Y.G. Shreter. Appl. Phys. Lett., 96, 133502 (2010).

[27] Н.И. Бочкарева, В.В. Вороненков, Р.И. Горбунов, А.С. Зубрилов, Ю.С. Леликов, Ф.Е. Латышев, Ю.Т. Ребане, А.И. Цюк, Ю.Г. Шретер. ФТП, 44, 822 (2010).

[28] M. Osinski, J. Zeller, P.-C. Chiu, B.S. Phillips, D.L. Barton. Appl. Phys. Lett., 69, 898 (1996).

[29] Н.И. Бочкарева, А.М. Иванов, А.В. Клочков, В.А. Тарала, Ю.Г. Шретер. Письма в ЖТФ, 42 (22), 1 (2016).

[30] M.J. Kappers, T. Zhu, S.-L. Sahonta, C.J. Humphreys, R.A. Oliver. Phys. Status Solidi C, 52 (4-5), 403 (2015).

Редактор Г.А. Оганесян

\section{Effect of deep centers on carrier confinement in InGaN/GaN quantum wells and efficiency of light-emitting diodes}

\author{
N.I. Bochkareva, Y.G. Shreter \\ loffe Institute, \\ 194021 St. Petersburg, Russia
}

\begin{abstract}
Deep center assisted tunneling of carriers in the $p-n$-structures of light-emitting diodes with InGaN/GaN quantum wells leads to a decrease in the effective height of the injection barrier but causes the dependence of radiation efficiency on the density and energy spectrum of defects in GaN. In the case of hopping conduction through the space charge region the main part of forward voltage drops near the quantum well boundary, where the density of deep states at the quasi Fermi-level is the lowest. As a result, the band bending at the border decreases, and with increasing current the direction of the electric field changes, which leads to weakening of the confinement of holes, their non-radiative recombination in the $n$-barrier and the efficiency droop. The low efficiency of green GaN LEDs is associated with the dominance of deep centers and insufficient density of shallow centers in the energy spectrum of defects in the barrier layers near the boundaries with quantum well. The proposed model is confirmed by the stepwise experimental dependences of the current, capacitance and efficiency of green and blue LEDs on forward bias reflecting the contribution of the color centers responsible for the defect photoluminescence bands in GaN.
\end{abstract}

